\title{
Autonomous Aerobraking Using Thermal Response Surface Analysis
}

\author{
Jill L. Prince ${ }^{1}$ and John A. Dec ${ }^{2}$ \\ NASA Langley Research Center, Hampton, Virginia, 23681 \\ and \\ Robert H. Tolson ${ }^{3}$ \\ North Carolina State University, Hampton, Virginia, 23666
}

\begin{abstract}
Aerobraking is a proven method of significantly increasing the science payload that can be placed into low Mars orbits when compared to an all propulsive capture. However, the aerobraking phase is long and has mission cost and risk implications. The main cost benefit is that aerobraking permits the use of a smaller and cheaper launch vehicle, but additional operational costs are incurred during the long aerobraking phase. Risk is increased due to the repeated thermal loading of spacecraft components and the multiple attitude and propulsive maneuvers required for successful aerobraking. Both the cost and risk burdens can be significantly reduced by automating the aerobraking operations phase. All of the previous Mars orbiter missions that have utilized aerobraking have increasingly relied on onboard calculations during aerobraking. Even though the temperature of spacecraft components has been the limiting factor, operational methods have relied on using a surrogate variable for mission control. This paper describes several methods, based directly on spacecraft component maximum temperature, for autonomously predicting the subsequent aerobraking orbits and prescribing apoapsis propulsive maneuvers to maintain the spacecraft within specified temperature limits. Specifically, this paper describes the use of thermal response surface analysis in predicting the temperature of the spacecraft components and the corresponding uncertainty in this temperature prediction.
\end{abstract}

\section{Nomenclature}

$\begin{array}{ll}\mathrm{A} & =\text { spacecraft area } \\ \mathrm{a}_{\mathrm{y}} & =\text { spacecraft acceleration in y direction } \\ \mathrm{AB} & =\text { aerobraking } \\ \mathrm{ABM} & =\text { aerobraking maneuver } \\ \mathrm{C}_{\mathrm{y}} & =\text { coefficient of drag - spacecraft y coordinate } \\ \mathrm{LMO} & =\text { low Mars orbit } \\ \mathrm{LMST} & =\text { local mean solar time } \\ \mathrm{m} & =\text { spacecraft mass } \\ \mathrm{MGS} & =\text { Mars Global Surveyor } \\ \mathrm{MRO} & =\text { Mars Reconnaissance Orbiter } \\ \mathrm{Q}_{\mathrm{max}} & =\text { maximum heat rate } \\ \mathrm{RS} & =\text { response surface } \\ \mathrm{V} & =\text { spacecraft velocity } \\ \rho & =\text { atmospheric density }\end{array}$

\footnotetext{
${ }^{1}$ Aerospace Engineer, Exploration Systems Engineering Branch, MS 489, Hampton, VA 23681; AIAA Member.

${ }^{2}$ Aerospace Engineer, Structural and Thermal Systems Branch, MS 431, Hampton, VA 23681; AIAA Member.

${ }^{3}$ Langley Professor, Mechanical and Aerospace Engineering Dept. 100 Exploration Way, Hampton, VA 23666, Associate Fellow.
} 


\section{Introduction}

Aerobraking is a proven method for increasing payload delivered to LMO. Typical mass saving is equivalent to over $1000 \mathrm{~m} / \mathrm{s}$ propulsion mass at Mars orbit insertion. After establishing a high eccentricity, long period orbit, aerobraking reduces orbital period and eccentricity to a desired science orbit by passing through the upper atmosphere 300 or more times. The limiting factor for past aerobraking missions has been the maximum temperature limit of the solar arrays, which are the main drag surfaces. Aerobraking has been used successfully for the following NASA spacecraft currently orbiting Mars: Mars Global Surveyor, Mars Odyssey, and Mars Reconnaissance Orbiter. Because of orbit to orbit atmospheric density variation, the temperature of the solar arrays can not be accurately predicted. At Mars, the average 1-sigma orbit-to-orbit density variation ${ }^{1}$ over the previous missions has been $40 \%$. Depending on latitude and season the orbit to orbit variability can range from $15 \%$ to $100 \%$. Consequently, the aerobraking operations phase has required many teams (navigation, atmospheric scientist, mission designers, spacecraft, thermal analyst, etc) to constantly monitor the mission throughout the aerobraking phase which can last from 3 to 6 months. Automating this process reduces workload, cost, and risk of potential human error. Automation may also increase aerobraking mission flexibility in that maneuvers can be chosen that are outside of a predetermined menu of maneuvers that are loaded on the spacecraft periodically ${ }^{2}$ rather than relying on ground-based personnel to choose a maneuver limited to optimal times within a common work-day. In addition, all aerobraking operations have used surrogate variables (maximum dynamic pressure or maximum free stream heat flux) for mission control in lieu of the real constraint, solar array temperatures.

After the MGS aerobraking experience, which spanned 15 months and over 800 aerobraking passes, it became clear that building more autonomy into aerobraking could significantly reduce mission cost. Three, increasingly more capable, approaches to automating the aerobraking operations phase were subsequently developed to adjust the flight sequence based on density inferred by accelerometer measurements during the aerobraking pass ${ }^{3,4}$. The time to initiate the aerobraking sequence is important because the spacecraft must be oriented to aerobraking attitude prior to atmospheric entry and reorient to cruise attitude after exiting the atmosphere. Due to the large variability in the Martian atmospheric density, mapping along track position becomes unacceptable uncertain after a few orbits. These methods essentially shift the time of periapsis of subsequent passes based on the measured drag during a pass. A similar method was tested and validated during Odyssey aerobraking ${ }^{2}$ but was not used operationally. This validated method was successfully implemented during MRO to shift the maneuver sequence ${ }^{5}$.

During the Mars Odyssey mission, which used a much more aggressive strategy aerobraking than MRO, extensive thermal analyses were performed daily to predict the solar array temperature based on the free stream heat flux from prior orbits. These predictions were compared with measured temperatures to validate and tune the preflight thermal models and confirm maneuver boundaries. The weak link in the process was the model used for the Martian atmosphere. The model had to be calibrated using radio tracking data and the heat flux predicted by the model had to be decreased by as much as $60 \%$ to be compatible with navigation results. Even so, the model could still not capture the actual shape of the density profile for many orbit passes (see figure 1) or the orbit to orbit variability which varied between $10 \%$ inside the polar vortex to $40 \%$ at lower latitudes. This figure presents the atmospheric density recovered from the accelerometer data ("Accel”) and the scaled model used by the project ("Mars-GRAM"). ${ }^{6}$ Heat flux is proportional to density times the velocity cubed. Nevertheless, aerobraking operations continued to use maximum heat flux as the control variable.

An approach that used measured solar array temperatures as the control variable was proposed as a means of removing the dependence on surrogate variables ${ }^{4}$. This approach proposed using an empirically derived relationship between measured solar array temperature and maximum heat flux during operations. A predictive filter was proposed to make the decision on whether to perform orbital maneuvers to either raise or lower periapsis based only the filtered heat flux. Also work was begun on using response surfaces to relate heat flux to maximum temperature and this approach was used successfully during MRO (ref). The purpose of this paper is to integrate the thermal response surface methodology with previous autonomous aerobraking algorithms in order to completely eliminate the dependence on surrogate variables. 


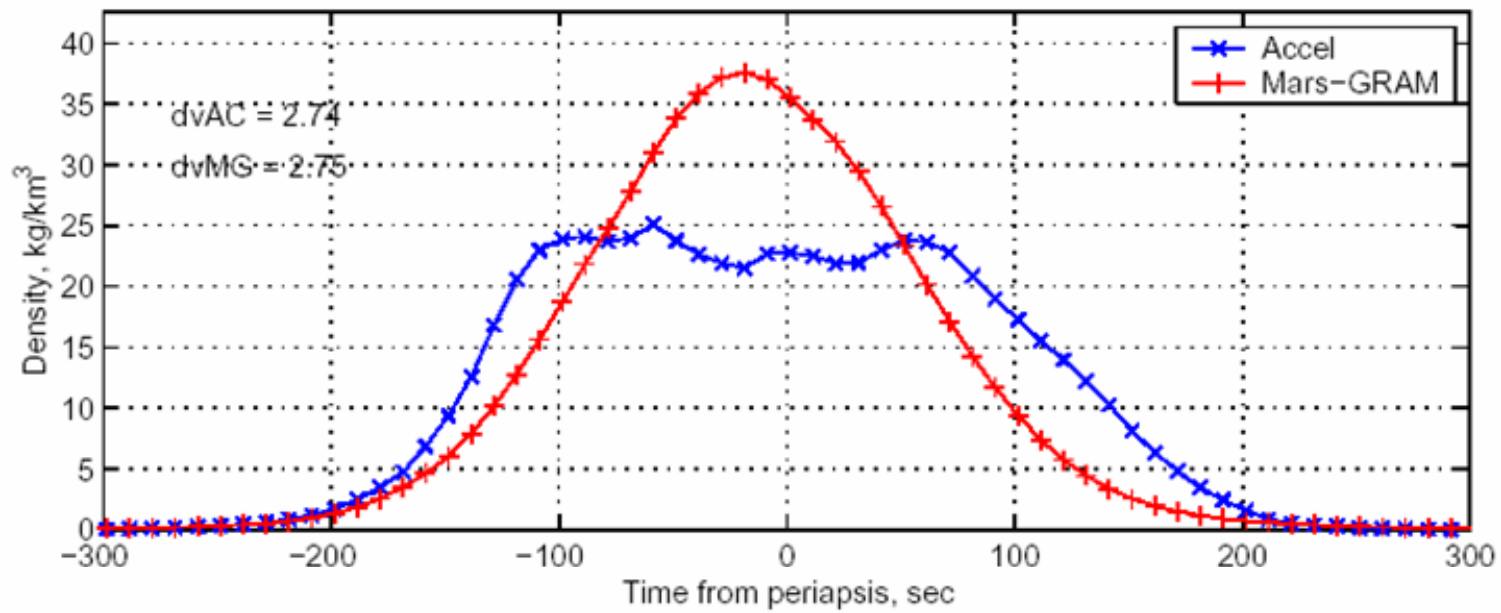

Figure 1. Odyssey periapsis 157 density profile.

\section{Mars Aerobraking Design}

The three Mars aerobraking missions to-date have flown the aerobraking phase with an upper and lower boundary or "corridor" on dynamic pressure or heat rate on the solar panels. Mars Global Surveyor was required to use dynamic pressure as the corridor upper limit because of the damage to the solar panel during the cruise phase. The dynamic pressure on the panel was limited to ensure no further damage during the aerobraking phase ${ }^{7}$. Both Mars Odyssey in 2001 and Mars Reconnaissance Orbiter (Figure 2) in 2006 used a heat rate corridor upper limit. This heat rate limit was used as the surrogate variable for making maneuver decisions to determine whether to increase atmospheric density and hence $\mathrm{Q}_{\max }$ by lowering periapsis altitude or conversely raise periapsis altitude and reduce $\mathrm{Q}_{\max }$. Heat rate was used because it could be derived from onboard accelerometer measurements or estimated from the orbit determination process. Further, $\mathrm{Q}_{\max }$ is a convenient variable for mission simulations since there was no direct method to correlate temperature predictions to predictive simulation techniques. Prior to aerobraking operations, the temperature limit of the solar panels was correlated to a heat rate limit for use by the flight mechanics simulations On all three missions the lower corridor limit was set by the mission requirement to end aerobraking with an orbit having a specified local solar time at the ascending node of the orbit. This constraint

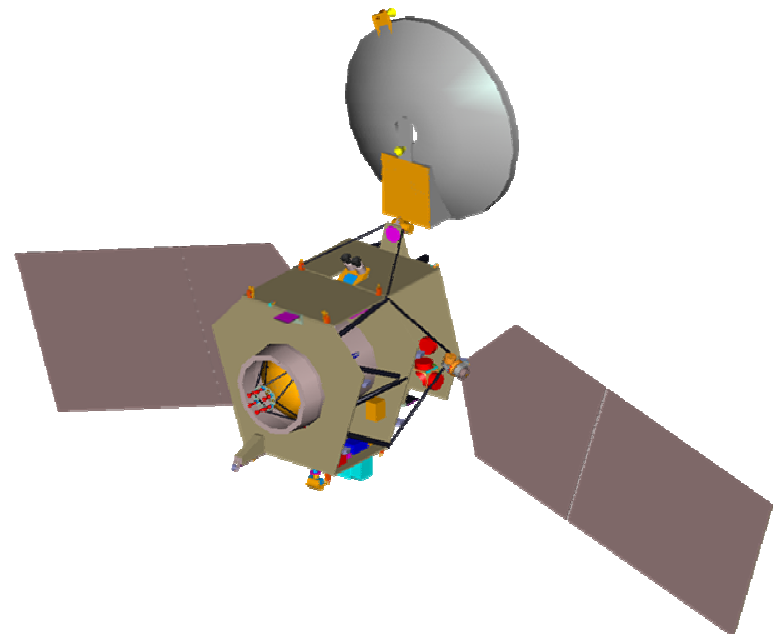

Figure 2. MRO in aerobraking configuration. essentially defined the day on which aerobraking had to end.

The flight qualification limit for the spacecraft solar panels was $195^{\circ} \mathrm{C}$. The flight allowable thermal limit was set at $175^{\circ} \mathrm{C}$ for Mars Odyssey and MRO to provide a safety margin. The conversion from this temperature limit to a $\mathrm{Q}_{\max }$ limit requires an assumed heating profile. The Mars density profiles were represented using a traditional isothermal atmosphere with a Gaussian uncertatinty distribution. The upper heat rate limit for MRO was set such that the nominal profile would complete aerobraking at 3:00 local mean solar time $(\mathrm{LMST})^{8}$. The lower boundary was set by other mission considerations such as number of maneuvers required to stay in the corridor and orbit geometry at the end of the $\mathrm{AB}$ phase. Because of the LMST requirement, the thermal constraints on the spacecraft were not restricting and MRO could fly to lower temperatures than Odyssey. A typical temperature distribution from the thermal model of the MRO solar panel is shown in figure 3. The red portions of the solar panel image represent the warmest temperatures that the 
solar panel withstood for this drag pass (periapsis 55), and in this instance, the maximum solar array temperature was estimated to be approximately $8^{\circ} \mathrm{C}$. The maximum temperature that the MRO solar panels experienced during the entire mission was estimated to have been approximately $40^{\circ} \mathrm{C}$. The maximum temperature during the Odyssey mission occurred on the anomalistic orbit 106 and was estimated at $135 \mathrm{deg}^{9}$.

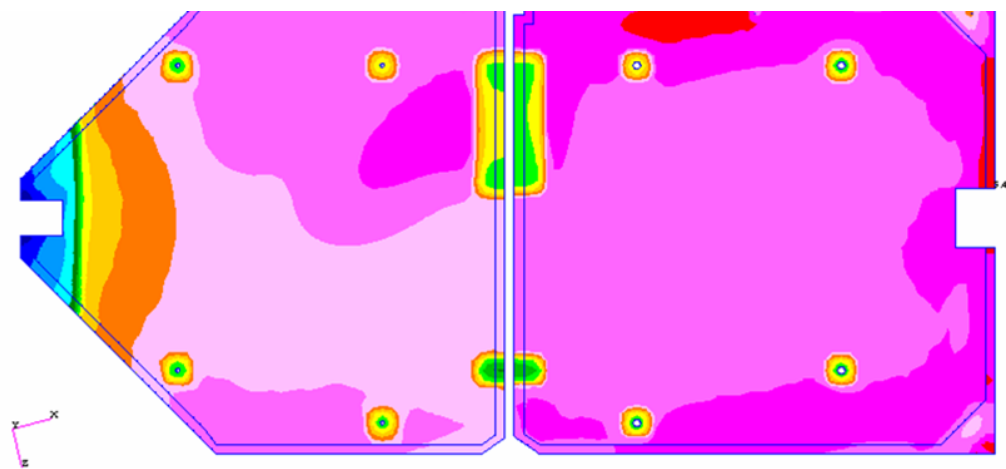

Figure 3. MRO solar array temperatures for periapsis 55.

III. Autonomous Aerobraking with Heat Rate Constraint

For autonomous aerobraking, a simulation has been designed to predict ahead a few orbits to determine what effects the Mars gravity perturbations and drag will have on subsequent orbits and whether or not a maneuver is needed. Solar pressure and gravity perturbations may also be required in the actual implementation onboard the spacecraft. Inclusion of such terms is straight forward and not considered any further here. It is assumed that the onboard ephemeris will be occasionally updated by the Navigation team; however, no estimates are made herein of the required update frequency.

For fully autonomous aerobraking, the onboard measurements of acceleration during the drag pass will be used directly in the equations of motion. Consequently, no global atmospheric model will be necessary. However, to predict drag for subsequent orbits, a model is required for maneuver decision purposes. In this process, accelerometer data are converted to atmospheric density $(\rho)$ via equation 1 assuming a constant drag coefficient, Cy, and a least square fit to $\log (\rho)$ versus altitude provides density at periapsis and local scale height.

$$
m a_{y}=\frac{\rho V^{2} C_{y} A}{2}
$$

These two parameters are then used to predict density for subsequent orbits (equation 2). In addition, a sliding least square fit using these density and scale height values would be used to determine any wave structure in the atmosphere $^{10}$. In the simulations, a simple atmospheric model with linear scale heights over specified altitude ranges is used along with the wave model and statistics from reference 10. These statistics account for all of the processes discussed above. Hence, in the simulations, density is modeled as

$$
\rho(h, \phi, \lambda)=\rho_{o}(h, \phi)\left[A_{o}+S(t)+\sum_{1}^{3} A_{n} \sin \left(n\left(\lambda-\lambda_{n}\right)\right)\right]
$$

where $\rho_{o}$ is a function of altitude and latitude from the linear scale height model. $A_{0}$ is a random variable with mean 1 and standard deviation between 0.15 and $0.19 .^{10}$ The randomness in $\mathrm{A}_{\mathrm{o}}$ accounts for unmodeled variations in the atmosphere and errors due to the density and scale height estimation process. S is included to simulate a dust storm. During MGS a regional dust storm caused density to increase by more than a factor of two in a few orbits. Any onboard autonomous algorithm will have to account for such storms. The remaining term represents three stationary waves. Amplitude and phases are given in reference 10 and except for the storm term, the model is the same as that given in reference 10.

A simulated example of autonomous aerobraking using a similar heat rate corridor to what was designed for MRO aerobraking is shown in figure 4. The red symbols indicate where an apoapsis propulsive maneuver (ABM) has occurred. This simulated mission is similar to actual flight operations. During MRO aerobraking operations, 26 maneuvers occurred in the 145 days of aerobraking, maintaining approximately $150 \%-250 \%$ margin.. This simulation produces 17 maneuvers in 145 days of aerobraking. Note that maneuvers in the autonomous aerobraking 
simulation are performed solely on the basis on the heating corridor. Lifetime constraint and collision avoidance issues are not taken into account here but
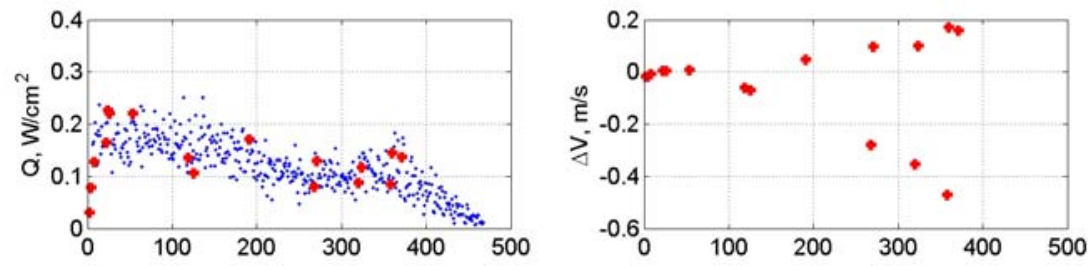

will be necessary in actual flight autonomy.

\section{Thermal Response Surface Analysis}
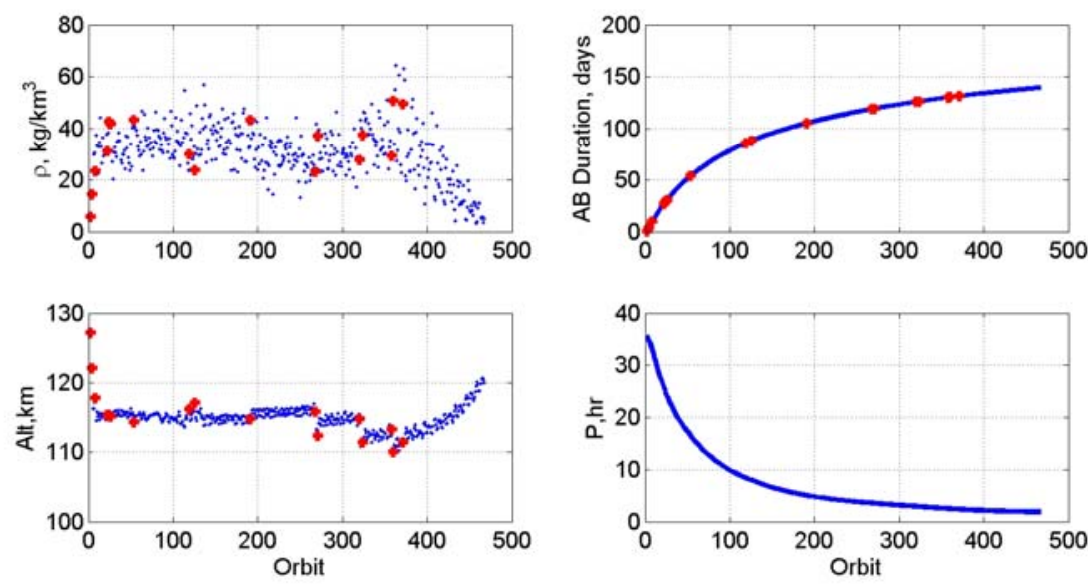

Figure 4. Simulation results from a heat-constraint autonomous aerobraking strategy.

\section{A. Thermal Response Surface Model}

A response surface is a simplified empirical model generally based on a more complex numerical model, in this case, a high fidelity finite element thermal model. In this implementation, the response surface is a quadratic equation which is derived from the finite element model $^{11}$. Using the design of experiments technique $^{11}$, the finite element model is systematically run whereby each run is performed using a different set of analysis input parameters. The analysis input parameters used are those assumed to be the most significant, or most influential in the analysis. In the case of the thermal analysis of the solar panels, variables fall into three categories, 1) environmental, such as the maximum atmospheric density, orbital period, and spacecraft velocity, 2) material property, such as M55J graphite composite face sheet emissivity, and 3) modeling variables, such as mass distribution. Using standard regression techniques, the coefficients of the response surface equation are determined, where the regression data are the temperature results from the finite element thermal model runs. The response surface equation is then just simply a quadratic equation for the peak solar panel temperature that is a function of the significant analysis parameters. For an operational aerobraking spacecraft, the only parameters that would vary orbit to orbit would be the environmental inputs. The response surface equation however, is only valid for a single discrete point on the solar panel. To track different locations, like the locations of the thermal sensors on the solar panel, multiple response surface equations are required. The advantage is clear, the response surface is a simple equation that can be evaluated in seconds as opposed to the finite element thermal model which takes an hour or more to run. Therefore, when given inputs such as max density, orbital period, and spacecraft velocity, it can be used to evaluate the temperatures of the solar panels of a given drag pass, in real time on board the spacecraft. The calculation speed also allows a Monte Carlo simulation to be performed in real time. With the Monte Carlo simulation results, for a given orbit, a 3 sigma temperature bound can be estimated and the probability of reaching the solar panel temperature limit can be calculated.

Thermal response surface analysis was first used for a spacecraft orbiting Mars during the Mars Reconnaissance Orbiter mission ${ }^{11}$. The analysis was validated during the aerobraking operations phase of the MRO mission and this analysis was later verified against the 2001 Mars Odyssey aerobraking phase. An example of the MRO thermal analysis using response surface is shown below. Figure 5 shows that given a very short calibration period of just a couple of orbits, the response surface can predict the temperature of the solar panel very accurately. For the 27 orbits shown, all flight data from the spacecraft were within 15 degrees of the response surface prediction and were well within the 3-sigma prediction of the temperature on the solar arrays. Figure 5 is an example of a prediction of the 
thermocouple T0110. Several discrete thermocouples were placed on the solar panel covering various temperature regimes on the front and back sides of the panel. All thermocouples as well as the maximum predicted temperature of the entire solar panel (not necessarily at a thermocouple location) were modeled and predicted throughout the

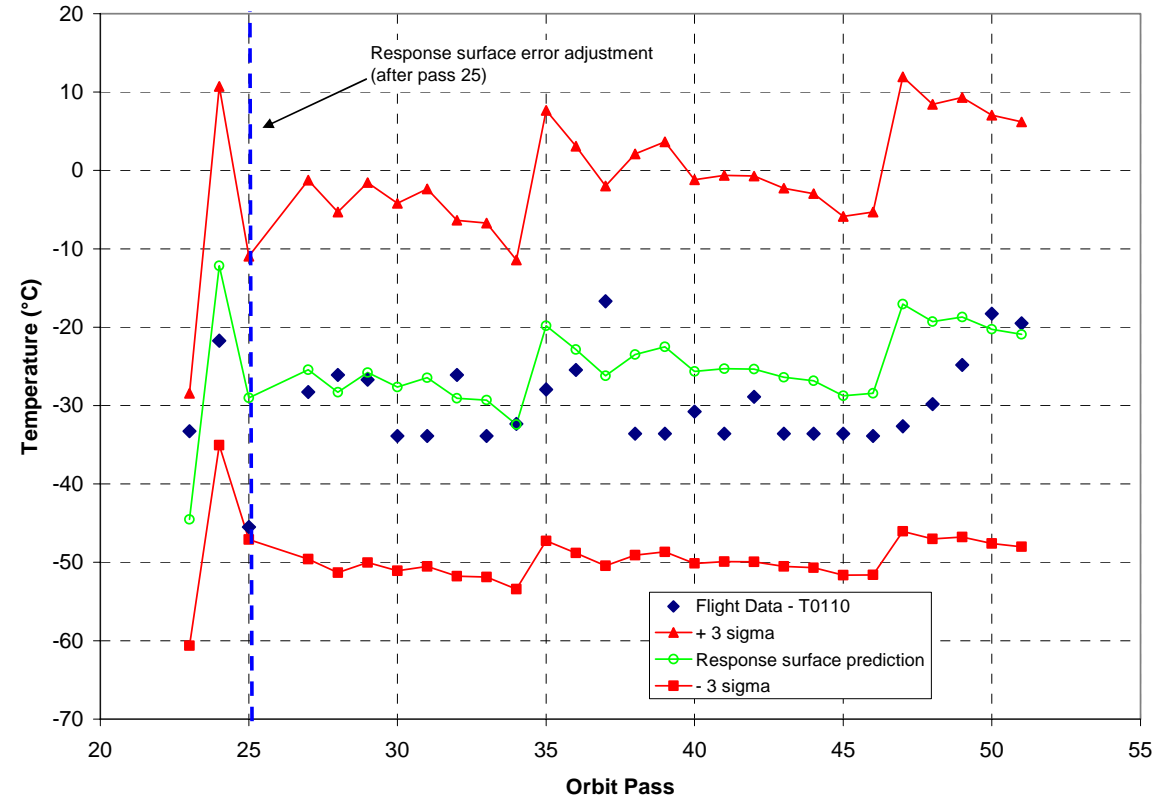

Figure 5. Comparison of response surface temperature predictions with flight data duration of the aerobraking phase.

Prediction of the solar panel temperatures is a key element required for autonomous aerobraking. In earlier studies $^{3}$ it was shown that with a low order gravity field, numerical integrator, and an adaptive atmospheric density model the spacecraft can autonomously predict ahead a few orbits. Including the thermal response surface analysis allows autonomous direct prediction of the temperature of the spacecraft to within a few degrees. In addition, the RS permits prediction of the uncertainty in the temperature. The spacecraft can then use a temperature corridor, rather than the dynamic pressure or heat flux corridor of previous missions. A temperature corridor more directly limits the vehicle based on the actual vehicle constraint: the temperature of the most limiting component, which for all the previous aerobraking missions was the solar panels.

The thermal response surface analysis was performed during aerobraking flight operations, but was not incorporated into trajectory simulations. Trajectory data were output to the thermal team at NASA Langley and then integrated into the thermal model ${ }^{12}$. The thermal response analysis was used to estimate the maximum temperature of the solar panels and determine how close to the thermal limit that the estimated 3-sigma high temperature would be. Analysis of mission statistics was performed on a weekly basis. An example of the operational use of the thermal response surface is shown in figure 6 . 


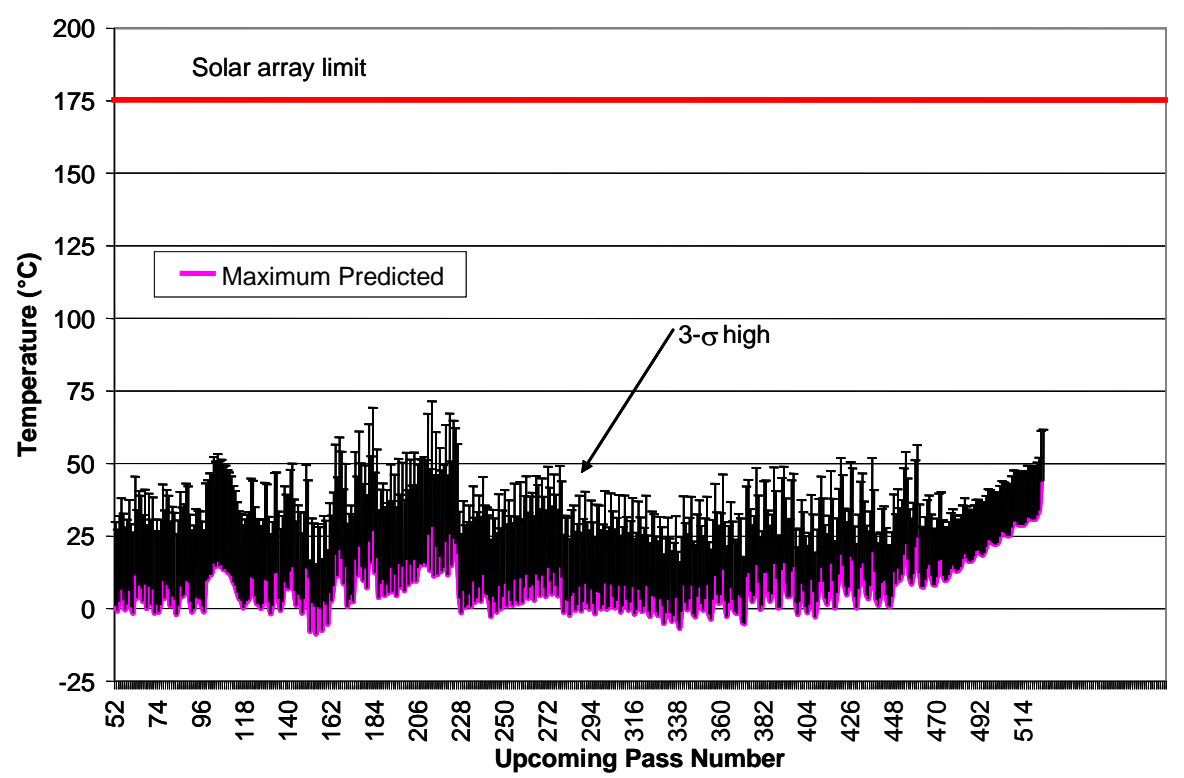

Figure 6. Predicted MRO solar array maximum temperatures from response surface analysis.
The magenta lines on this prediction represent the maximum predicted temperature on the MRO solar array. The black error bars show the 3sigma high error estimate from the response surface analysis. This particular analysis started from periapsis 52 and indicated that the maximum plus 3-sigma temperature was less than $75^{\circ} \mathrm{C}$ - which resulted in more than $150 \%$ thermal margin on the spacecraft. The thermal limit line of the solar panel is constant at $175^{\circ} \mathrm{C}$ as shown by the red line.

\section{B. Autonomous Aerobraking Simulation using Thermal Response Algorithm}

The previously described autonomous aerobraking simulation was enhanced by the addition of the thermal response surface algorithm. The analysis that was used to model the temperature of the solar panels during the MRO aerobraking phase was incorporated into the autonomous prediction capability of the simulation. Using the atmospheric density prediction described previously, a calculation for the drag duration, and the predicted period of the spacecraft, the maximum temperature of the solar panels is predicted for the subsequent 3 drag passes. The mean temperature prediction is used to evaluate the current and future position with respect to the defined temperature corridor. If outside the corridor, a single deterministic thermal response run is used to determine the desired density that would place the spacecraft to the desired temperature. A maneuver is performed at apoapsis based on the desired density and the predicted density. This maneuver logic is used throughout the simulated aerobraking phase and maintains temperatures similar to those seen during the MRO aerobraking phase. The results of this maneuver strategy using the thermal response surface algorithm are shown in figures 7 and 8. 

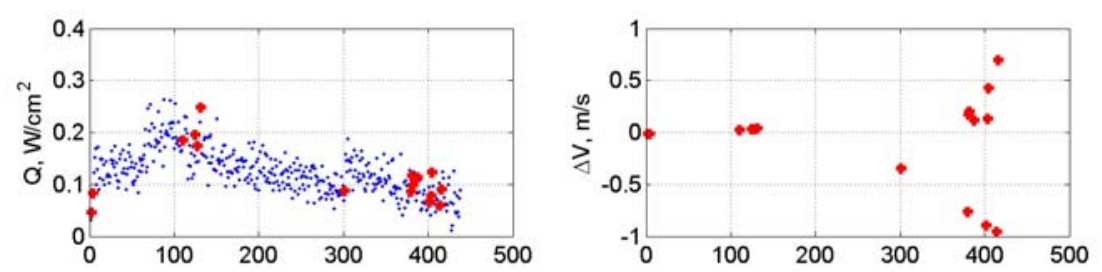

The spacecraft
panel temperatures shown in figure 3 illustrate the large amount of margin on the solar panels that was exercised during MRO aerobraking

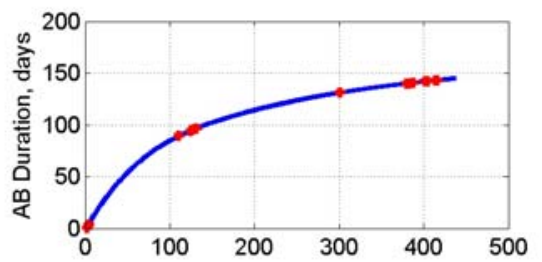

operations
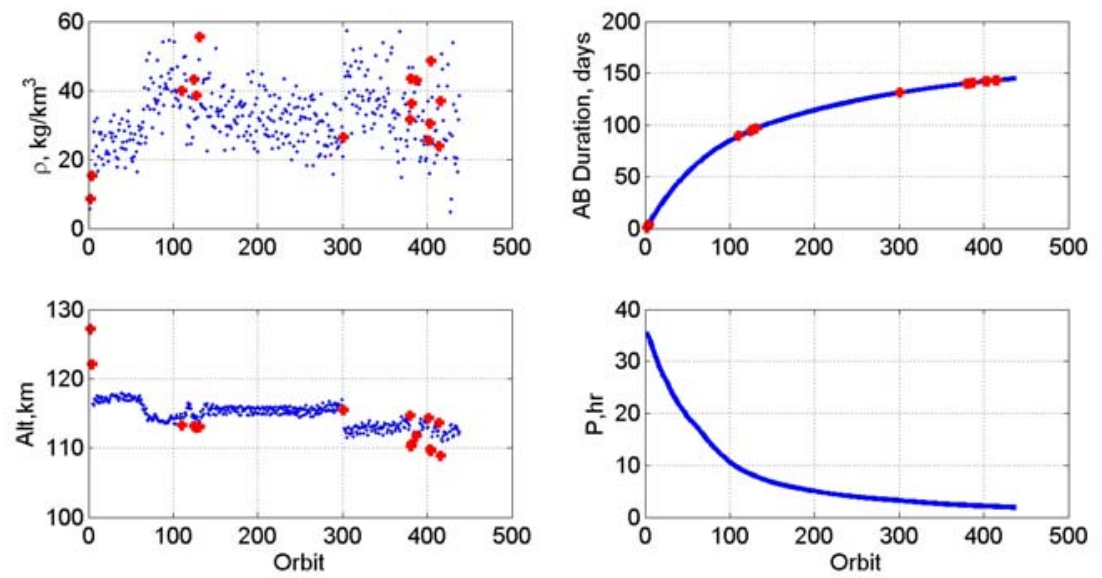

Figure 7. Simulation results from a thermal response surface autonomous aerobraking strategy.

(approximately 150250\%). This large margin was desired to reduce risk during the aerobraking phase since the science orbit required a 3:00 LMST. If this requirement were not applied to the MRO mission, the aerobraking phase may have been reduced and margin would have been smaller. It can be seen in figure 8 that the maximum temperatures of the solar array do not exceed approximately $50^{\circ} \mathrm{C}$, and the 3-sigma high estimates of predicted temperature do not exceed approximately $85^{\circ} \mathrm{C}$, well below the flight allowable limit of $175^{\circ} \mathrm{C}$. As in figure 4 , the red symbols indicate where an ABM has occurred to raise or lower periapsis altitude.

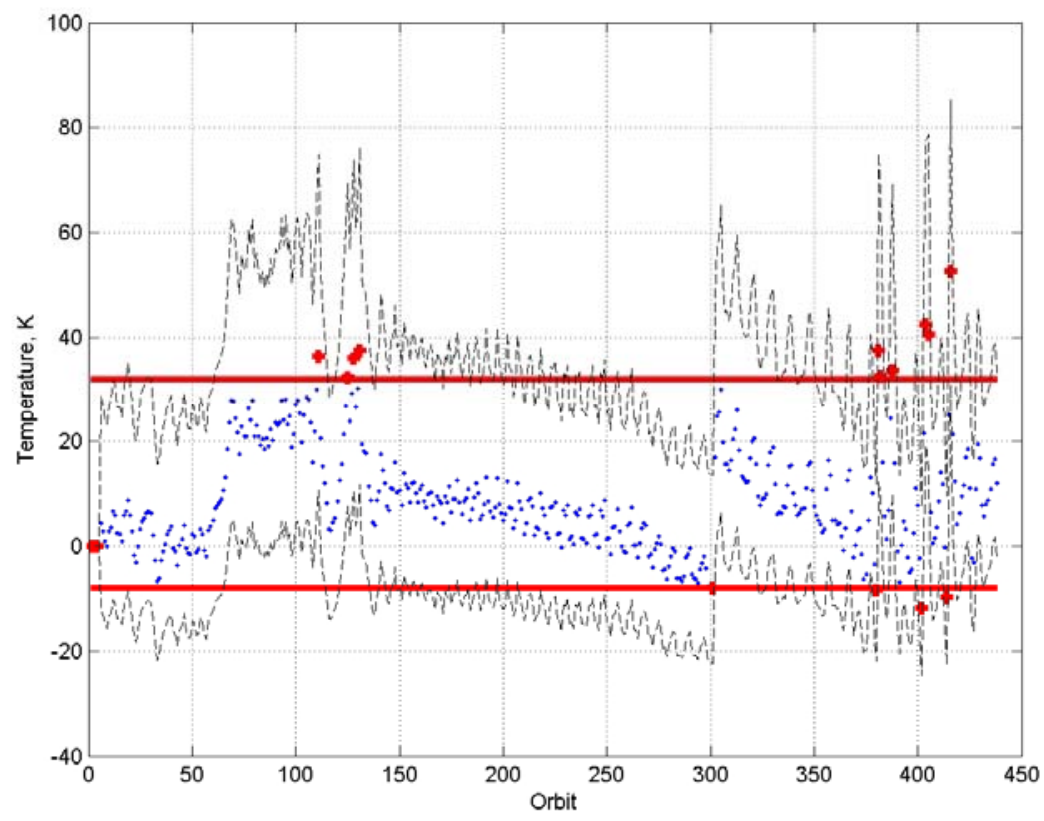

Figure 8. Temperature profile of autonomous aerobraking simulation using thermal response surface analysis. 
To emphasize the reduction in aerobraking duration that flying a reduced margin would allow, the autonomous aerobraking simulation was performed such that margin was reduced. Results are illustrated in figure 9 and show that the duration of the aerobraking phase while maintaining 200\% margin (from the nominal predicted temperature) is 100 days. Likewise, aerobraking duration is 89 days for $150 \%$ margin and 73 days for $100 \%$ margin. Even in the $100 \%$ margin case, the 3-sigma high temperature on the solar panel does not exceed the flight allowable limit of $175^{\circ} \mathrm{C}$. Again, lifetime constraint and collision avoidance maneuvers are not taken into consideration in this simulation. Actual durations may be slightly longer due to the reduction of heating necessary towards the end of aerobraking to avoid violation of the lifetime constraint.
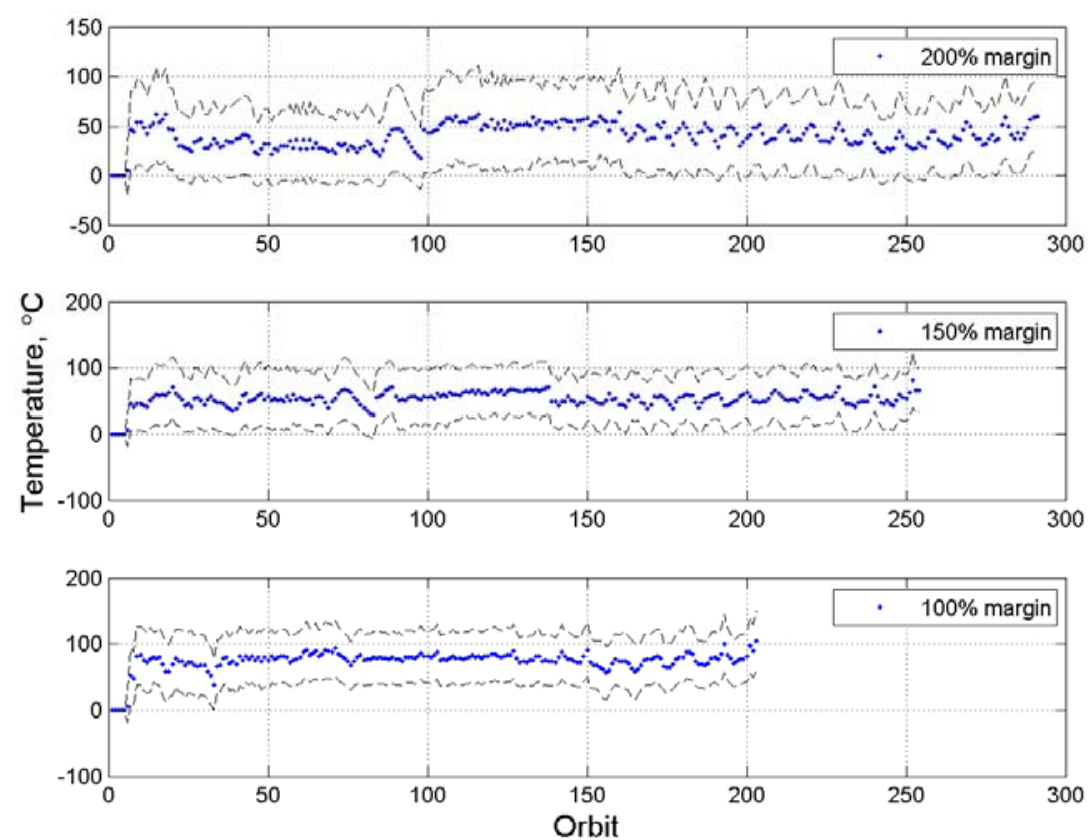

Figure 9. Effect of reduced thermal margin on aerobraking duration.

\section{Conclusion}

The use of autonomous aerobraking can significantly decrease workload, operations staff, and cost of aerobraking missions. Using the thermal response surface approach to determine the limiting constraint temperature - on the spacecraft, a more direct method of controlling the spacecraft is feasible. It has been shown during MRO aerobraking operations that the thermal response surface analysis determines solar panel temperatures accurately and consistently. This quick and effective tool is convenient and valuable in facilitating methods for autonomy in aerobraking operations.

\section{References}

${ }^{1}$ Tolson, R. H., et al., "Atmospheric Modeling Using Accelerometer Data During Mars Reconnaissance Orbiter Aerobraking Operations," AAS/AIAA Space Flight Mechanics Meeting, Sedona, AZ, Jan. 28-Feb. 1, 2007.

${ }^{2}$ Willcockson, W. and Johnson, M., "Mars Odyssey Aerobraking: The First Step Towards Autonomous Aerobraking Operations” 2003 IEEE Aerospace Conference, Big Sky, MT, March 9-14, 2003.

${ }^{3}$ Hanna, J.L. and Tolson, R.H., “Approaches to Autonomous Aerobraking at Mars” The Journal of the Astronautical Sciences, Volume 50, \#2, April-June 2002.

${ }^{4}$ Hanna, J.L., Tolson, R.H., Cianciolo, A.M.D, and Dec, J.A., “Autonomous Aerobraking at Mars”, $5^{\text {th }}$ International ESA Conference on Guidance Navigation and Control Systems and Actuator and Sensor Product Exhibition, Frascati, Italy, October 22-25, 2002. (ESA SP-516, February 2003)

${ }^{5}$ Johnson, Mark. Lockheed Martin Space Systems Corporation, private comm.

${ }^{6}$ Tolson, R. H., et al. “Application of Accelerometer Data to Mars Odyssey Aerobraking and Atmospheric Modeling” Journal of Spacecraft and Rocket. Vol.42 ,No.3, May-June, 2005. pp.435-443

American Institute of Aeronautics and Astronautics 
${ }^{7}$ Tolson, R.H., Keating, G.M., Cancro, G.J., Parker, J.S., Noll, S.N., and Wilkerson, B.L. “Application of Accelerometer Data to Mars Global Surveyor Aerobraking Operations”. Journal of Spacecraft and Rockets. Vol. 36, No. 3, May-June, 1999. pp. 323-329.

${ }^{8}$ Johnston, M. D., Graf, J. E., Zurek, R. W., Eisen, H. J., and Jai, B. “The Mars Reconnaissance Orbiter Mission”. IEEAC paper \#1174 2004 IEEE Aerospace Conference Proceedings

${ }^{9}$ Dec, J., et al. "Thermal Analysis and Correlation of the Mars Odyssey Spacecraft's Solar Array During Aerobraking Operations”. AIAA 2002-4975, Astrodynamics Specialist Conference, Monterey, CA August 5, 2002.

${ }^{10}$ Dwyer, A.M., Tolson, R.H., Munk, M.M., Tartabini, P.V. "Development of a Monte Carlo MarsGRAM Model for Mars 2001 Aerobraking Simulations”. The Journal of the Astronautical Sciences, Volume 50, \#2, April-June 2002.

${ }^{11}$ Dec, J. A., "Probabilistic Thermal Analysis During Mars Reconnaissance Orbiter Aerobraking", AIAA-2007-1214, Aerospace Science Meeting and Exhibit, Reno, NV, January 8-12, 2007.

${ }^{12}$ Prince, J.L. H., Striepe, S. A., “NASA Langley Simulation Capabilities for the Mars Reconnaissance Orbiter”, $15^{\text {th }}$ Annual AAS/AIAA Space Flight Mechanics Conference, Copper Mountain, Colorado, January 23-27, 2005. 